\title{
Feature-Cut: Video Object Segmentation Through Local Feature Correspondences
}

\author{
Dan Ring and Anil Kokaram \\ Sigmedia, Dept. of Electronic and Electrical Engineering \\ Trinity College, Dublin \\ dan@unworkable.org, anil.kokaram@tcd.ie
}

\begin{abstract}
Accurately segmenting objects in video is a difficult and time consuming process in modern post-production houses. Automatic systems may work for a small number of frames, but will typically fail over longer video shots. This work proposes a semi-automatic, feature-based system to perform object segmentation over longer sequences. The user manually extracts masks from representative instances of the object, which are then propagated to the remaining unsegmented frames and used to bootstrap the automatic segmentation for these frames. The presented work dramatically reduces the manual workload required to segment a video sequence, allowing longer and more accurate object mattes.
\end{abstract}

\section{Introduction}

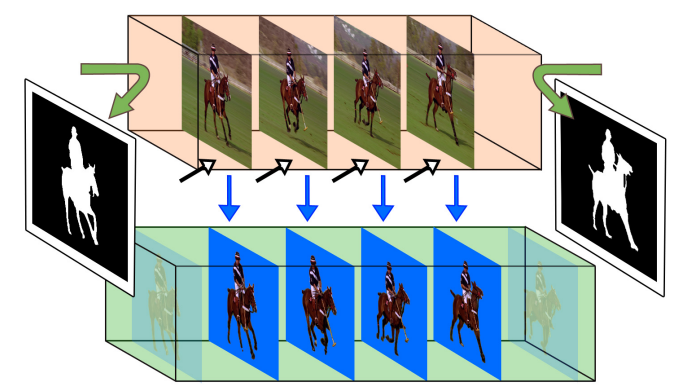

Figure 1. The objective of this work. The user supplies initial object mattes for a few frames, in this case 2 (black and white, far left and right). Information from these mattes is propagated using feature points, allowing the object to be quickly and accurately segmented in other frames automatically (bottom). This approach dramatically reduces the manual effort required to segment an object in video.

The task of extracting objects from video is known by many names, such as "video segmentation", "alpha mat- ting", "roto-scoping", etc. Essentially, they all arise from the same need; separating the image into object and notobject. In media production, video compositing workflows rely on the artist's ability to accurately select and extract objects from shots of video. To assist the artist, various semi-automatic video segmentation schemes exist, "autocompleting" the repetitive parts of the manual object cutout. The idea is to intelligently use the information supplied by the user in one frame to automatically segment other frames. However, to date no technique is robust enough to propagate information over more than a few frames. The novelty of the presented work lies in exploiting feature-based object detection research to propagate usersupplied segmentation information through video over long sequences, an example is shown in Figure 1.

\subsection{Previous Work}

Video segmentation is a well-studied area of research, resulting in a variety of applications and techniques. To put the presented work in context and provide focus to the reader, a selection of research relevant only to the postproduction application of segmentation is given.

The "Grab-Cut" algorithm by Rother et al. [13] developed a concise, effective method for segmenting images with the help of simple user strokes. The probabilistic framework used by Rother et al. allows additional feature spaces such as shape and motion to be sensibly incorporated. Similar research such as "Interactive video cutout" by Wang et al. [15], "Distance-Cut" by Bai \& Sapiro [16] also use user strokes to build a colour likelihood model of the object and background, yet extends the work of Rother et al. beyond a single image frame to a $3 \mathrm{D}$ volumetric space composed of the video frames over time. Bai \& Sapiro realised that using Graph-Cuts (as in [13] and [15]) for video segmentation are slow and do not scale well to segmenting long sequences. Instead, Bai \& Sapiro use the lineartime geodesic distances [17] to solve the labelling, allowing longer sequences to be segmented. This important strand 
of research resulted in the first usable forms of accurate, interactive video segmentation. Later, Criminisi et al. [5] developed this idea of using geodesic distances as an image filter for still image and video segmentation.

In practical examples of [15], [16] and [5], the algorithm works well over a few frames, but will begin to fail as the unseen data departs from the the user supplied information, requiring additional user interaction. The accuracy of the automatic portion of the algorithm and the frequency of the manual corrections depends on how much the chosen segmentation feature space (i.e. colour, shape, contour, geodesic distance etc.) varies over time. Sparse Features such as Harris-Laplace [9] and SIFT [11] are highly invariant to various photo-metric and geometric images conditions, allowing them to be reliably re-detected throughout a video sequence. This paper proposes using local features to propagate user supplied information further throughout the sequence.

Given a user created object matte in one frame, it is logical to use the apparent motion to propagate the mask to neighbouring frames, as shown by Gu \& Lee [10], and Choi et al. [4]. The problem with using motion to propagate information is the accumulation of errors over time. When the motion estimation process fails, the automated segmentation process fails too, and the user is back to manual correction. However, by establishing feature point correspondences between frames, it is possible to "pull" information from user supplied mattes into frames to be segmented. Unlike typical dense motion field matte propagation, feature correspondences can be identified between non-consecutive frames, allowing the propagation of user information from several temporally disparate frames at a time.

Section 2 describes the propagation of mattes using feature correspondences, Section 3 gives video object segmentation results from this system, with a discussion given in Section 4.

\section{Feature-Cut - Feature Based Matte Propa- gation}

The frames of a video to be segmented are divided into two sets, the set of frames already segmented by the user $S$, and the set of frames to be segmented $T$. The goal is to use the matte information from the frames in $S$ to segment each frame in $T$. For each frame $t \in T$, matte information from $S$ is then "pushed" along feature correspondences between the frame $t$ and the frames in $S$. The question now is how the matte information should be propagated?

The work of Marszalek et al. [12] uses the idea of "shape masks" to detect and localise objects within a candidate image. This involves matching features between images in a database and the image to be localised. For each feature match between an image pair, the feature locations are used

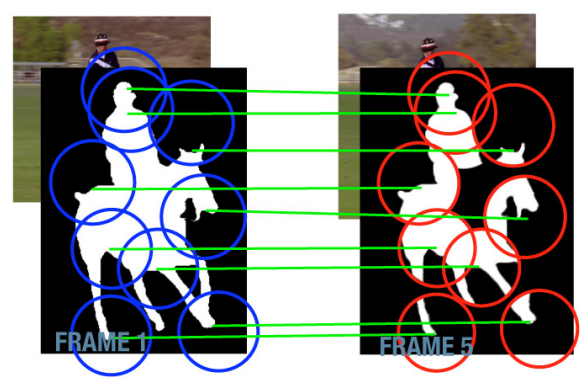

Figure 2. Example of propagating manually segmented matte information from frame 1 to frame 5 of the "Polo" sequence. Using feature correspondences between the frames (green), pieces of the matte are pushed into frame 5. Each of the blue \& red circular pieces represents the image region of the SIFT feature descriptor used to identify the match. Notice that the partial matte in frame 5 is missing information, and does not entirely delineate the object. However, there is still enough useful information in the partial matte to allow a more accurate object matte be refined.

to "vote" for the location of the object in the candidate image. A well-localised object will have many votes and appear as a well-defined object "mask". Feature-Cut extends the idea of shape masks for localisation to the task of accurate object segmentation as follows, an example illustrating the idea is shown in Figure 2. Initial putative feature correspondences are first identified by the "nearest-neighbour" in the feature's SIFT descriptor [11] space. Matches are rejected if the distance between the pair of descriptors is above a value $d_{t}$. The remaining matches are refined further by applying the spatial consistency constraint of Sivic et. al., requiring that at least $k$ similar feature correspondences lie in the vicinity of a potential match [14]. This spatial constraint is both fast and reliable at removing typical bad matches. For the presented results, $d_{t}$ and $k$ were set to 0.4 and 2 respectively ${ }^{1}$.

The fact that a correspondence has been made between a pair of images indicates that the image content between the images at the sites of the matched feature points is similar. As shown by the work of Marszalek et al. [12], it makes sense that the matte around the feature in frame $s \in S$ can be "transplanted" onto the corresponding region in the tobe-segmented frame, $t \in T$. The actual "transplant" operation between two image patches is defined by the scale $\mathbf{D}$ and rotation $\mathbf{R}$ transforms between the pair of matched features. The matrices $\mathbf{D}$ and $\mathbf{R}$ are simply by the change in

\footnotetext{
${ }^{1}$ Assuming the SIFT descriptors are normalised such that the $L^{2}$ norm of each descriptor is 1 , the range of distance values between a pair of descriptors is $[0, \ldots, \sqrt{2}]$.
} 
the canonical feature scales and rotations:

$$
\begin{aligned}
& \mathbf{D}=\left[\begin{array}{cc}
\left(\frac{\rho_{p}}{\rho_{q}}\right) & 0 \\
0 & \left(\frac{\rho_{p}}{\rho_{q}}\right)
\end{array}\right] \\
& \mathbf{R}=\left[\begin{array}{cc}
\cos \left(\theta_{q}-\theta_{p}\right) & \sin \left(\theta_{q}-\theta_{p}\right) \\
-\sin \left(\theta_{q}-\theta_{p}\right) & \cos \left(\theta_{q}-\theta_{p}\right)
\end{array}\right]
\end{aligned}
$$

where $\rho_{p}$ and $\rho_{q}, \theta_{p}$ and $\theta_{q}$, are the canonical scales and angles of the feature points $p$ and $q$ respectively. The matrix $\mathbf{u}$ defines the relative sites the image patch, $\mathbf{u} \in[-r, \ldots, r] \times$ $[-r, \ldots, r]$, where $r$ is the radius or extent of the patch being transplanted, and is set to the radius over which the SIFT descriptor is calculated, typically $3 \rho_{q}$. Given a source matte $M(\mathbf{x})$ from frame $s \in S$, the transformed matte using a single feature match $(p, q)$ in the image patch $\mathbf{u}$ is defined as:

$$
M^{\prime}(\mathbf{q}+\mathbf{u})=M(\mathbf{p}+\mathbf{D R u})
$$

Given that the image content between the feature pair $(p, q)$ is already similar, the resultant transplanted matte should appear reasonable in the context of the new image, yet is probably not perfect. To help correct potentially incorrect matte pixels, the warped region matte $M^{\prime}(\mathbf{q}, \mathbf{u})$ is weighted by the pixel similarity of the warped matched pixel regions ${ }^{2}$ ,$\Delta I=1-\left\|I_{t}(\mathbf{q}+\mathbf{u})-I_{s}(\mathbf{p}+\mathbf{D R u})\right\|$. This is the propagation of matte information for a single feature match.

Consider now, extending this idea to include all the feature matches $(p, q)$ between $s$ and $t$, call this set $N_{(s, t)}$, for all the source frames $s \in S$. As in [12], $M_{f}^{\prime}(\mathbf{q})$ and $M_{b}^{\prime}(\mathbf{q})$ are the summations of the transplanted object and not-object partial mattes over all matches, defined as:

$$
\begin{aligned}
& M_{f}^{\prime}(\mathbf{q}+\mathbf{u})=\sum_{s \in S} \sum_{(p, q) \in N_{(s, t)}} g(\mathbf{u}, r) M_{s}(\mathbf{p}+\mathbf{D R u}) \Delta I \\
& M_{b}^{\prime}(\mathbf{q}+\mathbf{u})=\sum_{s \in S} \sum_{(p, q) \in N_{(s, t)}} g(\mathbf{u}, r)\left(1-M_{s}(\mathbf{p}+\mathbf{D R u})\right) \Delta I
\end{aligned}
$$

$M_{f}^{\prime}(\mathbf{q})$ and $M_{b}^{\prime}(\mathbf{q})$ can be thought of as a "vote-space", where the values of $M_{f}^{\prime}$ and $M_{b}^{\prime}$ at a pixel site $\mathbf{x}$ are the number of features that "voted" for the pixel $\mathrm{x}$ to be object or not-object respectively. $g(\mathbf{r}, \mathbf{q})$ is a circular window function of radius $r$, where $g(\mathbf{u}, r)$ is 1 if $\|\mathbf{u}\| \leq r$ and 0 otherwise.

As the source matte $M_{s}$ is a binary matrix, the accumulation of pixels labelled not-object (a matte value of 0 ), would always equal 0 . Therefore, object and not-object matte information needs to be propagated seperately, resulting in

\footnotetext{
${ }^{2}$ For simplicity It is assumed here that the maximum value of $\| I_{t}(\mathbf{q}+$ $\mathbf{u})-I_{s}(\mathbf{p}+\mathbf{D R u}) \|$ in this case is 1 . However, for most practical cases this will need to be changed to suit the actual values of $I$.
}
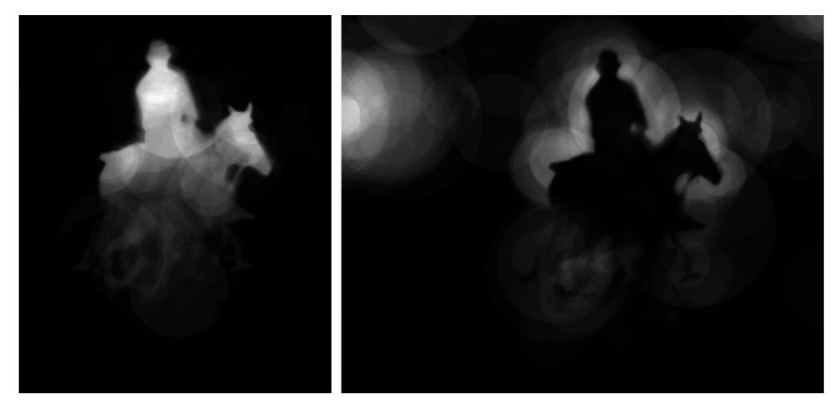

Figure 3. Example of propagated matte information from three matte source frames. The accumulated partial mattes of the object $M_{f}^{\prime}(\mathbf{q})$ and $M_{b}^{\prime}(\mathbf{q})$ background are shown on the left and right respectively. Brighter regions are the result of more feature matches in the region. Although there is relatively less information in the horses legs, there is still enough to create an accurate matte.

$M_{f}^{\prime}(\mathbf{q})$ and $M_{b}^{\prime}(\mathbf{q})$. An example of both accumulated partial mattes is shown in Figure 3.

The quality of the propagated mattes relies on the number and quality of the feature correspondences. For example, consider the "horse and rider" object mattes in Figure 3. The horse's legs have relatively fewer matches than those for the horse's body and rider, and because of this, the mattes in Figure 3 are not as well defined for the legs. Thresholding the partial mattes as-is would not result in a useful matte. However, there is enough information provided by the partial mattes to extract a more accurate matte.

\subsection{Refining the Partial Matte}

Although the propagated mattes do a reasonable job of delineating the object, as seen in Figure 3, the mattes are far from useable. For example, relatively few matches were found on the horse's leg (Figure 3, left), which means there is simply no information to say whether the pixels in the region belong to the object or not. However, it is expected that the object / not-object labelling should be "smooth". That is, label information from nearby pixels can be taken into consideration to help label the difficult regions. Continuing the example of the horse's leg, it is sensible that the leg should be have the same label as the body. This implies a smoothness constraint on the label field. In our situation, we use the energy minimisation framework of Grab-Cut [13] to accurately segment the current frame. The same neighbourhood smoothness constraints are employed, and the propagated foreground and background partial mattes are used as the likelihood functions directly.

Consider the frame to be segmented as a vector of pixel at sites $\mathbf{x} \in\left\{\mathbf{x}_{\mathbf{1}}, \ldots, \mathbf{x}_{\mathbf{n}}, \ldots, \mathbf{x}_{\mathbf{N}}\right\}$ with values $\mathbf{z} \in$ $\left\{z_{1}, \ldots, z_{n}, \ldots, z_{N}\right\}$. The vector $\alpha \in\{0,1\}$ is the label vector assigning the pixel at site $x_{n}$ to background or object for $\alpha_{n}$ values of 0 and 1 respectively. The set of partial mat- 
tes for the frame is $M^{\prime}=\left\{M_{f}^{\prime}, M_{b}^{\prime}\right\}$. Label smoothness is enforced by a Gibbs energy function [7]. The modified energy function of Grab-Cut [13] is defined as follows:

$$
\mathbf{E}\left(\alpha, M^{\prime}, \mathbf{x}, \mathbf{z}\right)=U\left(\alpha, M^{\prime}, \mathbf{x}, \mathbf{z}\right)+\lambda V(\alpha, \mathbf{x}, \mathbf{z})
$$

where:

$$
\begin{aligned}
U\left(\alpha, M^{\prime}, \mathbf{x}, \mathbf{z}\right) & =\quad \sum_{n} \exp -\gamma\left(M_{f}^{\prime}\left(x_{n}\right)\right) \alpha_{n} \\
& +\quad \sum_{n} \exp -\gamma\left(M_{b}^{\prime}\left(x_{n}\right)\right)\left(1-\alpha_{n}\right) \\
V(\alpha, \mathbf{x}, \mathbf{z}) & =\sum_{(n, m) \in \mathbf{C}}\left\|x_{n}-x_{m}\right\|\left(\left|\alpha_{n}-\alpha_{m}\right|\right) w_{n, m}
\end{aligned}
$$

$U$ and $V$ are the data and spatial energy terms, with $\lambda$ being used to weight the contribution between the two. C is the set of pair-wise neighbourhood cliques between adjacent "compass-point" pixels. The function $w_{n, m}$ is used to weight the spatial energy term of a pair of pixels by the difference in their values, defined in this work as $w_{n, m}=$ $\exp -\beta\left(z_{n}-z_{m}\right)$. Intuitively, the spatial energy term $V$ penalises pixels having different labels than their neighbours. If the scalar weight $\beta$ is $0, V$ becomes the "Ising" prior, otherwise the neighbourhood penalty is weighted by how different the neighbouring pixel values are from the current pixel. This encourages smoothness of pixel labels except across edges. The scalar variable $\gamma$ is used to weight the contribution of the partial mattes. From [3], the variables $\beta$ and $\gamma$ are defined as:

$$
\begin{aligned}
& \beta=\left(2\left\langle\left(z_{n}-z_{m}\right)^{2}\right\rangle\right)^{-1} \\
& \gamma=\left(2\left\langle M^{\prime}(\mathbf{x})^{2}\right\rangle\right)^{-1}
\end{aligned}
$$

where $\langle$.$\rangle is the expectation operator over the whole image.$ By calculating $\beta$ and $\gamma$ in this way, $\beta$ is adjusted accordingly for high and low constrast images, and $\gamma$ is adjusted to accommodate the effects on the partial mattes from high and low numbers of feature points in the image. With the data and spatial energy terms introduced, the labelling of the current frame, $\hat{\alpha}$, is achieved by minimising the energy function $\mathbf{E}\left(\alpha, M^{\prime}, \mathbf{x}, \mathbf{z}\right)$ :

$$
\hat{\alpha}=\arg \min _{\alpha} \mathbf{E}\left(\alpha, M^{\prime}, \mathbf{x}, \mathbf{z}\right)
$$

The energy function is minimised using Graph-Cuts [8, 2]. The parameter $\lambda$ in Equation 1 determines the amount of influence pixels have upon their neighbours. This is vitally important in the proposed segmentation system, as it allows for data in regions of strong likelihood to affect regions with weaker or no likelihood energies, for example, regions where no feature matches have been found. For the presented results, a value of $\lambda=0.05$ was used for the "Polo" sequences, as the likelihood energies for pixels belonging to the horse's legs are not strong, and a high $\lambda$ value risks cutting them off. For the other sequences a value of $\lambda=0.1$ was used.

\section{Results}

To objectively compare the results from the presented algorithm, ground-truth mattes were created for four video sequences using the "Quick-Select" tool in Adobe Photoshop [1]. To focus the discussion of the results, only four "interesting" sequences are presented. The sequences chosen exhibit various photo-metric conditions that often make segmentation difficult, such as highly-textured scenery, motion blur, flat texture-less regions, and low colour contrast. For the purposes of these results, every 10th frame was manually segmented, and the automatically generated object mattes were not touched-up afterwards. The number of Hessian-Laplace features calculated per image was capped at 2000 per frame. Results and details of the sequences are presented in Table 1. The processing times given are from a $1.8 \mathrm{GHz}$ Intel Dual Core2 processor.

Table 1. Segmentation results compared to ground truth. $\bar{t}$ is the mean processing time per frame.

\begin{tabular}{|l|l|l|l|l|l|}
\hline Sequence & Resolution & $\begin{array}{l}\text { No. } \\
\text { frames }\end{array}$ & fps. & $\begin{array}{l}\text { Man. } \\
\text { frames }\end{array}$ & $\bar{t}$ \\
\hline Eating Apply & $640 \times 360$ & 100 & 15 & 10 & $5.4 \mathrm{~s}$ \\
Quadbike & $720 \times 576$ & 50 & 15 & 5 & $8.6 \mathrm{~s}$ \\
Polo & $720 \times 576$ & 100 & 15 & 10 & $6.0 \mathrm{~s}$ \\
Oktoberfest & $640 \times 480$ & 144 & 15 & 14 & $7.0 \mathrm{~s}$ \\
\hline
\end{tabular}

\subsection{Visual Evaluation}

For a visual comparison of results, example frames from the four sequences presented in Figures 4, 5, 6 and 7. Similar to the results in Table 1, every 10th frame was manually segmented as a source frame, and the automatically generated mattes are not touched-up afterwards. The compositing of the results onto the blue background was performed by using the results of the presented algorithm as input to the non-binary matting tool in Nuke [6]. A comparison between automatic Feature-Cut and manual segmentation algorithms is shown in Figure 10.

To illustrate the accuracy of the refined object matte, close-ups of frames from the "Eating Apple" and "Quadbike" sequences are shown in Figures 8 and 9. The close-up of Figure 8 highlights some interesting properties of the presented algorithm. The scene from "Eating Apple" contains a mixture of dense, detailed plant foliage and a "head-andshoulders" with little texture. This detailed scene is reasonably difficult for traditional segmentation features, such as colour or edges. However, stable feature points are generally detected around highly textured regions, and as the performance of the algorithm is related to the number of feature points, the more detailed and complex the scene the greater the matte accuracy. Notice in Figure 8 that the detail of the leaves is preserved, particularly in the branch occluding the 
subjects face. The close-up of the "Quad-bike" matte in Figure 9 also illustrates how difficult regions are successfully segmented, such as the sparse frame of the quad-bike.

The results of the "Oktoberfest" sequence shown in Figure 7 are interesting. The shot begins by a left-to-right pan of the crowd, then focuses on the subject for a while, and resumes the pan. The manually segmented frames containing the subject were taken from the middle of the shot. Notice in the top and bottom rows of Figure 7 (corresponding to the beginning and end of the shot), the subject is not in the shot, and is correctly not segmented. As the automatic segmentation process is based upon matching features between images, no explicit object detection is needed. With the presented algorithm, the object is free to enter and exit the shot, and will only be segmented when it is in the frame.

The segmentation results in Figures 11 and 12 show examples where the algorithm fails. In Figure 11, regions of the rider's arms disappear. In this case, the video frame is the last one in the sequence and is relying on the appearance of the arm to remain unchanged since the manually extracted matte information from 10 frames prior. There is also the problem of foreground and background colours merging, such as the handlebars. As these regions are relatively texture-less, few feature points are found there, making the segmentation more reliant upon the spatial prior than the data energy of Equation 1.

\subsection{Statistical Evaluation}

When comparing segmentation results to ground truth, error measures such as the Mean-Squared-Error (MSE) have limited meaning for two reasons: Firstly, segmentation is subjective, results that are acceptable for one user, may not be usable for another. Secondly, the ground truth is usually generated manually by a user, with subjective ideas of what pixels represent the object and background. From the first reason, it is difficult, if not impossible, to calculate a number that objectively measures how good the results of a segmentation are. Instead, it is more meaningful to say whether the results from the system differ from results that an average user would manually extract.

To evaluate this, ground truth is needed for each frame of every sequence from a reasonable number of users. This is an unreasonable amount of manual effort, and difficult to interpret when compared to the automatic segmentation results. Instead, for a single chosen frame in each sequence, other users are asked to extract the ground truth. The author extracts ground truth for each frame of each sequence will be extracted by the author and used as a reference. The idea is to first show that the reference ground truth is sufficiently similar to the other users ground truth for the selected frame of each sequence. If they are sufficiently similar for that one frame, it is assumed that all of the author's reference ground truth frames for all sequences are representative of typical

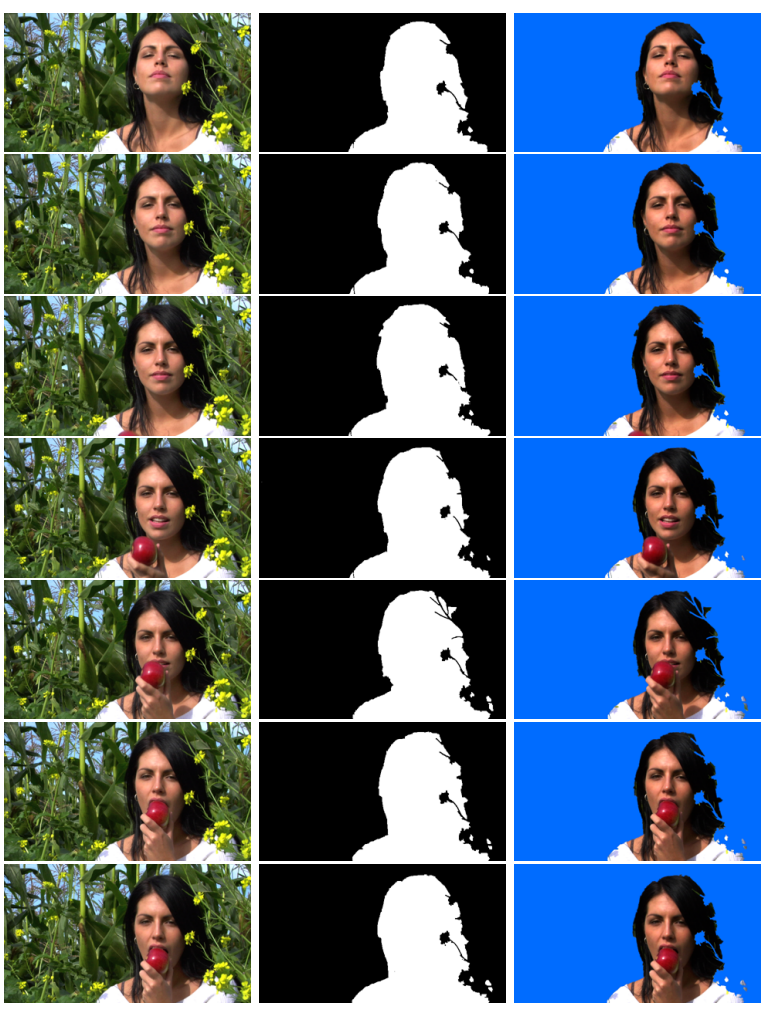

Figure 4. Results of the "Eating Apple" sequence. Original (left), Automatic Matte (centre) and Composite (right).

user's ground truth. Using the author's ground truth as reference, the automatic segmentation results can be tested to see whether the differences between algorithm and reference mattes are typical of differences between mattes from different users. Before beginning the evaluation, the mattes to be compared and their methods of comparison need to be defined.

To get an idea of how differently users select objects in an image, a frame from the sequence to be tested is manually segmented by $N$ different users, in this case $N=7$. The difference $\epsilon_{u}$ between two binary ground truth mattes (from users $i$ and $j$ ) $M_{i}$ and $M_{j}$, indexed by the set of pixel locations $X$, is given by $\epsilon_{u}(i, j)=\sum_{\mathbf{x} \in X} \mid M_{i}(\mathbf{x})-$ $M_{j}(\mathbf{x}) \mid$. The set of differences between user mattes is given by comparing each of the $N$ user mattes to the other mattes, for example, a matte from user $i$, is compared against all mattes from users $[i+1, \ldots, N]$, the matte from user $i+1$ is then compared to mattes from users $[i+2, \ldots, N]$, and so on, resulting in $\frac{N(N-1)}{2}$ values of $\epsilon_{u}$. Similarly, the difference $\epsilon_{s}$ between the author's ground truth matte $M_{s}$ and another user $i$ 's matte $M_{i}$ for the single frame is given by $\epsilon_{s}(i)=\sum_{\mathbf{x} \in X}\left|M_{s}(\mathbf{x})-M_{i}(\mathbf{x})\right|$. For a sequence of $F$ frames, the difference $\epsilon_{g}$ between the automatically generated mattes $M_{a}$ and the author's ground truth mattes $M_{g}$ for frame $f \in[1, \ldots, F]$ is given by 


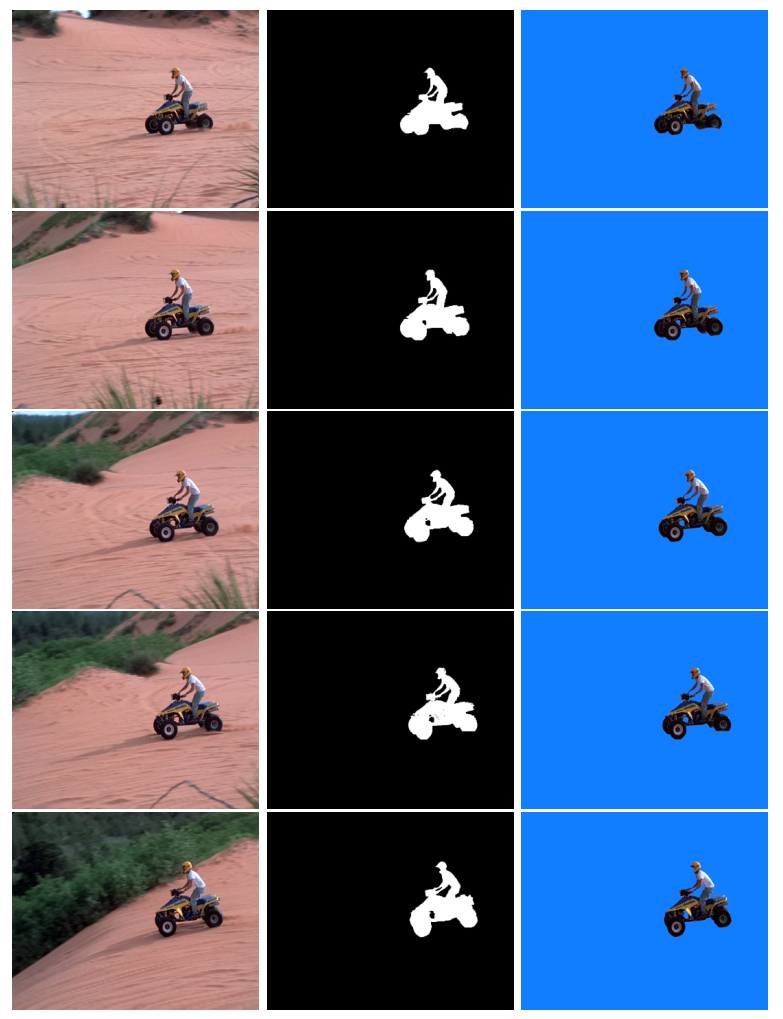

Figure 5. Results of the "Quad-bike" sequence. Original (left), Automatic Matte (centre) and Composite (right).

$\epsilon_{g}(f)=\sum_{\mathbf{x} \in X}\left|M_{a}(f, \mathbf{x})-M_{g}(f, \mathbf{x})\right|$.

In a statistical sense, two hypotheses need to be tested for each sequence. Firstly, it is asserted that the ground truths mattes of the author are not significantly different from ground truth mattes of other users, in terms of the null hypothesis, $H_{0}^{1}: \bar{\epsilon}_{u}=\bar{\epsilon}_{s}$. Secondly, given that the author's ground truth is representative of typical user mattes, it is asserted that the automatic segmentation results are not significantly different from the author's ground truth, $H_{0}^{2}: \bar{\epsilon}_{g}=\bar{\epsilon}_{u}$. However, $H_{0}^{2}$ only asserts that the distributions of $\epsilon_{g}$ and $\epsilon_{u}$ are sufficiently similar. In some cases, the distribution of differences between the automatically generated mattes and the author's ground truth mattes may be significantly lower. Therefore, an additional null hypothesis is tested, $H_{0}^{3}: \bar{\epsilon}_{g}<\bar{\epsilon}_{u}$. Paired Student's "t" tests are performed, and evaluated at the $\alpha=0.05$ significance level. The results for the "Polo", "Eating Apple" and "Quad-bike" sequences are presented in the following table. (For brevity, "acc." and "rej." indicates acceptance and rejection of the proposed hypothesis.)

The first interesting result to note, is that the null hypothesis $H_{0}^{1}$ asserting that the author's ground truth mattes are sufficiently similar to other user's ground truths is accepted. Had this been rejected, the ground truth manually created by the author would not be representative of typi-

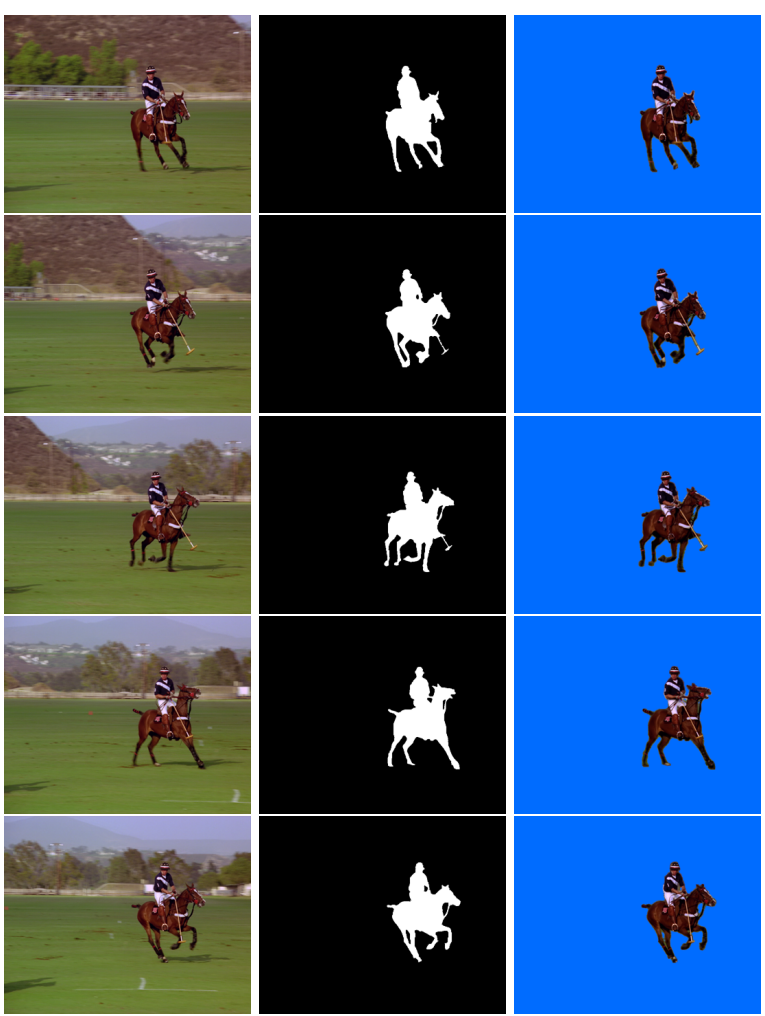

Figure 6. Results of the "Polo" sequence. Original (left), Automatic Matte (centre) and Composite (right).

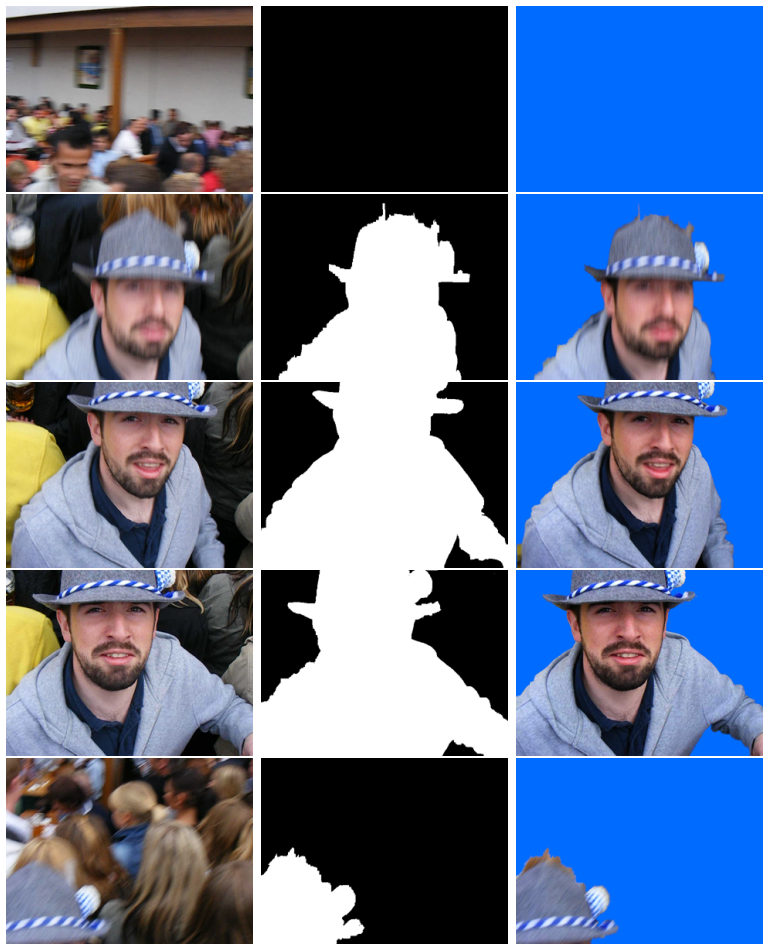

Figure 7. Results of the "Oktoberfest" sequence. Original (left), Automatic Matte (centre) and Composite (right). 

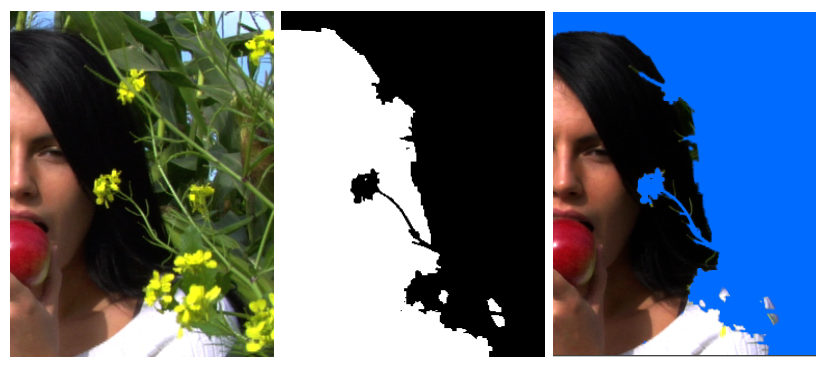

Figure 8. Close-Up of results from the "Eating Apple" sequence. From left to right; original, automatic matte and non-binary matte composite. Despite the intricate shapes of the plant's branches, an accurate matte is found. Notice however, that some finer plant branches were not segmented. During the manual segmentation of the object in other frames, the same fine structures were not selected by the user, and so have not been segmented by the algorithm. Whether strong fidelity to the user mattes is good or not is a matter of taste.
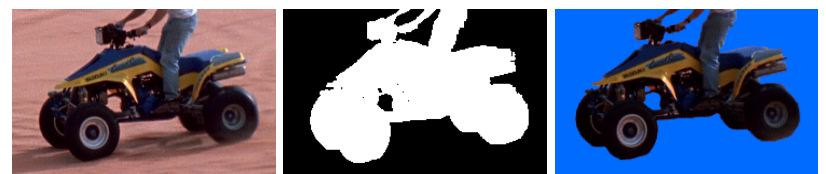

Figure 9. Close-Up of results from the "Quad-bike" sequence. From left to right; original, automatic matte and non-binary matte composite. Two things are interesting here; the see-through parts around the frame of the quad-bike are preserved, and foreground I background boundaries with low contrast (such as the shadows and wheels) are correctly and accurately segmented.

\begin{tabular}{|c|c|c|c|c|c|c|}
\hline Sequence & $H_{0}^{1}$ & $p_{0}^{1}$ & $H_{0}^{2}$ & $p_{0}^{2}$ & $H_{0}^{3}$ & $p_{0}^{3}$ \\
\hline Eating Apply & acc. & 0.47 & rej. & 0.0 & acc. & 1 \\
Polo & acc. & 0.14 & acc. & 0.1 & rej. & 0.052 \\
Quadbike & acc. & 0.74 & rej. & 0.018 & acc. & 0.99 \\
\hline
\end{tabular}

cal users, and useless as a basis for comparison. The next important result is that the null hypothesis $H_{0}^{2}$ asserting the differences between the automatic and author's ground truth mattes are consistent with the differences between typical user mattes is accepted for the "Polo" sequence. The reason that $H_{0}^{2}$ is rejected for the "Eating Apple" and "Quad-bike" sequences is that the differences between the automatic and author's ground truth mattes are significantly less than differences between typical user mattes, therefore accepting the null hypothesis $H_{0}^{3}$. Although $\bar{\epsilon}_{g}$ is lower than $\bar{\epsilon}_{u}$, this does not necessarily mean that the automatic segmentation results are better than mattes that a typical user would create. However, had $\bar{\epsilon}_{g}$ been significantly higher than $\bar{\epsilon}_{u}$, this would have indicated very poor mattes, and a complete failure of the segmentation system. Given that either $H_{0}^{2}$ or $H_{0}^{3}$ is accepted for all sequences, the results of the automatic segmentation algorithm are not significantly different

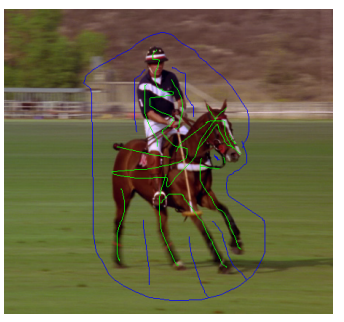

User strokes

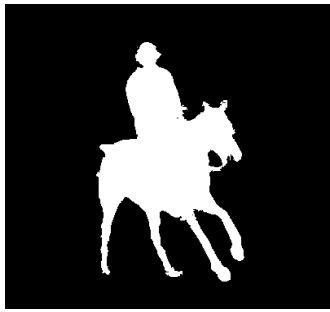

Distance-Cut

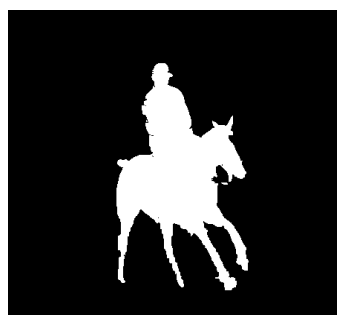

Feature-Cut

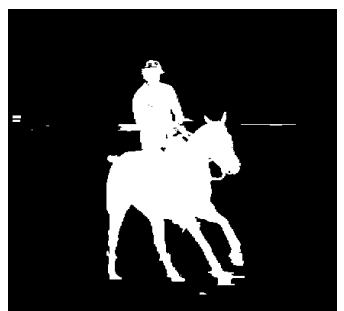

Grab-Cut
Figure 10. Comparison of the presented algorithm to other segmentation schemes. The Feature-Cut segmentation is the result of having segmented manually every 10th frame. This frame is from the middle of a pair of selections, i.e. frame 5. The foreground and background user strokes for Distance- and Grab-Cut are shown in the left image, as green and blue respectively. Notice that the results of the Feature-Cut segmentation are not significantly different from the manual Distance- and Grab-Cut segmentation algorithms, and in the case of Grab-Cut, slightly better.
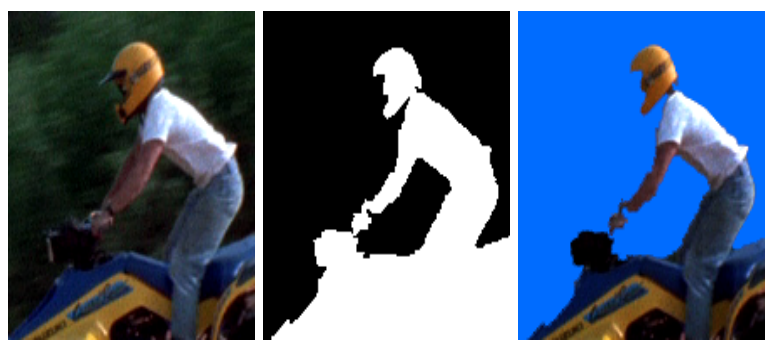

Figure 11. Example of the algorithm failing on the "Quad-bike" sequence. The lack of contrast between the background and parts of the rider and quad-bike have caused parts of the face, helmet, arms and handlebars to become "eroded".

from mattes that a typical user would manually create for the same sequences.

\section{Discussion}

This paper has presented a feature-based approach to video object segmentation that "pulls" user supplied matte information from temporally disparate regions in the video to the remaining un-segmented frames throughout the sequence. This greatly reduces the effort required by the artist to create a object matte throughout a video sequence, al- 

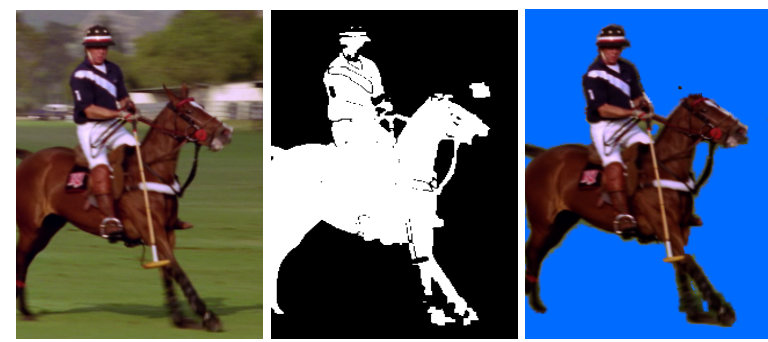

Figure 12. Example of the algorithm failing on the "Polo" sequence. Centre, the low number of feature correspondences between this frame and those with user-supplied mattes (mostly due to motion blur) has caused a number of missing regions on the horses legs and ears. Also, incorrect feature matches have introduced blotches falsely accepted as object, and labelled part of the helmet and jersey as being background. Notice that the non-binary matte (right) can compensate for a lot of mistakes, but the result is still not perfect.

lowing the user to segment longer videos, with higher accuracy. The results of Table 1 show that the manual effort required to segment the frames can be dramatically reduced by nearly an order of magnitude for comparable levels of quality. The proposed Feature-Cut system allows faster creation of accurate mattes over longer sequences.

The achilles heel of any segmentation scheme is the feature space used. For example, colour segmentation fails if the colour in the image is poor, contour segmentation fails if the edge information is poor, and motion segmentation fails if the apparent motion is poor. The main problem with feature-based segmentation is of course, when feature information is poor. Texture-less regions, motion-blur and non-uniform object deformation make it difficult to detect reliable features. These conditions are exemplified by the horse's leg in Figure 6. Although texture-less regions can be compensated for during refinement of the propagated partial mattes, there are some situations where the presented feature-based segmentation will fail. It may be possible to include additional user information in these cases, in a form other than matte information, to improve segmentation performance.

Another issue is that each frame to be automatically segmented is processed independently of consecutive frames. For example, following the manual segmentation stage, the remaining frames can be processed in any order; no temporal consistency is enforced. This has the effect of pixels "popping" in and out of the automatically generated mattes over time. In practice, the non-binary matte stage will generally be able to compensate and reduce the apparent effects. However, it makes sense to incorporate information between consecutive frames to produce better mattes before applying non-binary matting, and will be investigated in future work.

\section{Acknowledgments}

The authors would like to acknowledge and thank the After Effects and DMRG groups at Adobe Systems. This work was funded by the Irish Research Council for Science, Engineering and Technology (IRCSET) Embark Initiative, the i3DPost project under the EU Framework 7 ICT programme, and Adobe Systems.

\section{References}

[1] Adobe Systems Incorporated. Adobe CS4 Photoshop Manual, 2008.

[2] Y. Boykov, O. Veksler, and R. Zabih. Fast approximate energy minimization via graph cuts. In $I C C V$, volume 1, pages 377-384, 1999.

[3] Y. Y. Boykov and M. P. Jolly. Interactive graph cuts for optimal boundary \& region segmentation of objects in n-d images. In ICCV, volume 1, pages 105-112, 2001.

[4] J. G. Choi and S. W. Lee. Semi-automatic video object segmentation method based on user assistance and object tracking. In Knowledge-Based Intelligent Information and Engineering Systems, volume 3214, pages 211-218, 2004.

[5] A. Criminisi, T. Sharp, and A. Blake. Geos: Geodesic image segmentation. In ECCV, volume 1, pages 99-112, 2008.

[6] The Foundry. Nuke Manual, 2008.

[7] S. Geman and D. Geman. Stochastic relaxation, gibbs distributions, and the bayesian restoration of images. TPAMI, pages 452-472, 1990.

[8] V. Kolmogorov and R. Zabih. What energy functions can be minimized via graph cuts? In TPAMI, volume 26 , pages 147-159, February 2004.

[9] C. S. Krystian Mik. Bernardiolajczy. Scale \& affine invariant interest point detectors. IJCV, 60(1):63-86, 2004.

[10] C. G. M.-C. Lee. Semiautomatic segmentation and tracking of semantic video objects. Circuits and Systems for Video Technology, IEEE Transactions on, 8:572-584, September 1998.

[11] D. G. Lowe. Distinctive image features from scale-invariant keypoints. IJCV, 60(2):91-110, November 2004.

[12] M. Marszałek and C. Schmid. Accurate object localization with shape masks. In CVPR, jun 2007.

[13] C. Rother, V. Kolmogorov, and A. Blake. "grabcut": interactive foreground extraction using iterated graph cuts. ACM Trans. Graph., 23(3):309-314, 2004.

[14] J. Sivic and A. Zisserman. Video google: A text retrieval approach to object matching in videos. In ICCV, page 1470 , 2003.

[15] J. Wang, P. Bhat, R. A. Colburn, M. Agrawala, and M. F. Cohen. Interactive video cutout. ACM Trans. Graph., 24(3):585-594, 2005.

[16] G. Xue Bai Sapiro. Distancecut: Interactive segmentation and matting of images and videos. In ICIP, volume 2, pages 249-252. IEEE, October 2007.

[17] L. Yatziv, A. Bartesaghi, and G. Sapiro. O(n) implementation of the fast marching algorithm. J. Comput. Phys. 212(2):393-399, 2006. 